\title{
Una propuesta metodológica para analizar la coherencia de políticas para el desarrollo
}

\author{
Natalia MiLlÁN ACEVEDO \\ Instituto Complutense de Estudios Internacionales \\ natalia.millan@icei.ucm.es
}

Recibido: 10-01-2014

Aceptado: 13-10-2014

\section{Resumen:}

El objeto del presente artículo es desarrollar una propuesta metodológica para analizar la Coherencia de Políticas para el Desarrollo (CPD). Con este propósito, en primera instancia se analizan las distintas visiones sobre la CPD para establecer luego un enfoque comprehensivo de Coherencia adaptado a los procesos de interdependencia y transnacionalización propios de la actual era global. En este marco, se propone un marco metodológico basado en cuatro dimensiones de análisis con una agenda de investigación para cada una de ellas. En cada dimensión se incorpora los ámbitos a investigar, los actores involucrados así como una serie de preguntas de investigación que pudieran valer de guía para el desarrollo de un estudio comprehensivo en CPD.

Palabras clave: Coherencia de Políticas; desarrollo humano; marco metodológico; dimensiones.

\section{A methodological framework for studying policy coherence for development}

\begin{abstract}
:
The purpose of this article is to develop a methodological framework for analyzing Policy Coherence for Development (PCD). With this goal, we analyze different views of PCD in order to establish a comprehensive approach of Coherence adapted to globalization process. In this context, the article proposes a methodological framework based on four dimensions of analysis in CPD. In each dimension, we set the areas and actors to investigate and we put forward research questions that could be use as a guide for a comprehensive approach to study PCD.
\end{abstract}

Keywords: Policy Coherence; Human Development; methodological framework; dimensions.

\section{Referencia normalizada}

Millán Acevedo, N. (2014): "Una propuesta metodológica para analizar la coherencia de políticas para el desarrollo", Política y Sociedad, 51 (3), pp. 673-693. 
Sumario: Introducción. 1. Una aproximación a la visión del desarrollo humano. 2. El concepto de Coherencia de Políticas para el Desarrollo. 3. Una propuesta teórica metodológica para un análisis comprehensivo de la CPD. 4. Una propuesta teórica metodológica para un análisis comprehensivo de la CPD. 5. Reflexiones finales. Bibliografía.

\section{Introducción}

Desde la década de los noventa, el concepto de Coherencia de Políticas para el Desarrollo (CPD) ha adquirido paulatinamente un mayor espacio analítico en los espacios académicos concernientes a los estudios de desarrollo. La creciente importancia del enfoque de la CPD radica en el hecho que para comprehender cabalmente la posible contribución de un Estado a la promoción de un mundo más justo y equitativo, es necesario valorar todas las políticas públicas que este Estado desarrolla así como las consecuencias que estas políticas tienen para otros países y personas, que es precisamente el objetivo de análisis de la CPD. De ahí que sea necesario superar el paradigma de análisis unidimensional que tradicionalmente es utilizado en las Relaciones Internacionales para desarrollar un enfoque comprehensivo que permita valorar el potencial papel de un Estado en la promoción y garantía de los derechos humanos en el mundo.

Además, fruto de los cambios estructurales generados para los progresivos y crecientes fenómenos de interdependencia y globalización, los procesos de desarrollo deben ser valorados cada vez más en función de las dinámicas globales, regionales y locales. De esta forma, los problemas del desarrollo ya no se encuentran "contenidos" o delimitados por el espacio territorial de un Estado nación, sino que se trata de desafíos que se han transnacionalizado. Así, la dinámica globalizadora ha cuestionado la capacidad real de los Estados para diseñar políticas y respuestas "nacionales" frente a los diferentes contextos, transformaciones y desafíos que se derivan de las diferentes coyunturas globales. En este contexto, la CPD se vuelve un factor fundamental en tanto revela las opciones y limitaciones que se presentan en el escenario internacional así como las interdependencias estatales en la promoción del desarrollo humano y global.

El presente artículo pretende delinear un concepto de CPD más adaptado a los cambios estructurales de la actual era global así como proponer un marco analítico comprehensivo de CPD para el estudio de las políticas públicas. Con este propósito, luego de esta introducción, el primer epígrafe establece el concepto de desarrollo humano sobre el que se asienta el concepto de CPD propuesto; el segundo epígrafe, analiza el concepto de CPD y sus implicaciones para la era global; para profundizar en el tercero epígrafe, sobre la propuesta metodológica para analizar la CPD desde un enfoque multidimensional. El artículo finaliza con algunas reflexiones sobre los cambios estructurales derivados del proceso de globalización y sus implicaciones para el estudio de la CPD. 


\section{Una aproximación a la visión del desarrollo humano}

El debate sobre la definición, alcance e implicaciones del desarrollo humano se ha convertido en uno de los elementos más fundamentales para los estudios sobre esta problemática. En sus orígenes, los llamados "pioneros del desarrollo"1 (1940-1950) relacionaban estrechamente este concepto con el crecimiento económico desarrollando diversas teorías sobre los problemas del desarrollo económico en los países más pobres. Desde esa época, ha existido tradicionalmente una tendencia a identificar el bienestar humano con el crecimiento económico como uno de los principales fundamentos teóricos y metodológicos de la ortodoxia económica (Unceta, 2009). Así, durante décadas se ha evaluado el desarrollo considerando sólo las actividades monetizables sobre otras consideraciones teóricas como pueden ser la sostenibilidad medioambiental, la equidad de género, la redistribución de la riqueza o la calidad de las instituciones (Unceta, 2009). Cabe destacar, además, que este enfoque de crecimiento está basado en patrones de consumo y producción ambientalmente insostenibles que ignoran los límites naturales del planeta (PNUD, 2010; Oxfam, 2011).

Sin embargo, a medida que los estudios de desarrollo fueron progresando y ampliándose se ha ido complejizando la definición de este concepto, incorporando elementos básicos para el desarrollo humano como es la sostenibilidad ambiental, la equidad de género, los derechos humanos o la redistribución de la riqueza, por sólo mencionar algunos ejemplos.

El concepto de desarrollo humano remite a la ampliación de las capacidades, libertades y opciones de todas las personas del planeta y de las futuras generaciones. En tal sentido, el premio Nobel de Economía, Amartya Sen, describe al desarrollo como libertad, libertad para que las personas puedan elegir cómo vivir sus vidas y, para ello, se debe garantizar a toda la ciudadanía el libre y pleno ejercicio de sus derechos (Sen, 1999). Esta visión del desarrollo es asumida, al menos en el ámbito discursivo, por buena parte del conjunto de actores estatales y multilaterales del sistema internacional de desarrollo.

"El desarrollo humano es aquel que sitúa a las personas en el centro y las hace protagonistas de su propio proceso de desarrollo, basándose en un enfoque de derechos humanos (...) Es la garantía del pleno ejercicio de los derechos fundamentales, pero también medios materiales, ingresos y riqueza, oportunidades, acceso a servicios de educación o salud, es poder expresarse en democracia y tener seguridad, po-

${ }^{1}$ Los "pioneros del desarrollo" son aquellos cuyos estudios sobre este tema llegaron a dominar el pensamiento sobre el desarrollo económico entre 1940 y 1960. Los autores más destacados en este aspecto han sido Lord Bauer, Colin Clark, Albert Hirschman, Sir Arthur Lewis, Gunnar Myrdal, Raul Prebisch, Paul Rosenstein-Rodan, Walt Whitman Rostow, H.W. Singer y Jan Tinbergen. Para mayor información, véase, Meyer, G. y D. Seers (1986): Pioneros del desarrollo, Banco Mundial. 
der vivir en un medio ambiente sano alrededor, y es también confianza y autoestima en el propio proyecto vital" (MAEC, 2013: 14).

Desde la perspectiva de este artículo, tres son los elementos que subyacen a esta definición de desarrollo humano:

En primer lugar, se trata de un concepto de desarrollo multidimensional que incorpora ámbitos imprescindibles de las organizaciones humanas. En este marco, las dimensiones sociales, ambientales, civiles, políticas y económicas se deben integrar de igual manera al trabajo por el desarrollo humano al tiempo que ninguna de estas puede ser priorizada sobre las demás. De este modo, si bien el crecimiento económico se presenta como una condición positiva que genera oportunidades, éste no parece ser condición suficiente para garantizar la ampliación de las libertades y opciones de las personas.

En segundo lugar, es una visión basada en un enfoque de derechos lo que supone un proceso de empoderamiento para las comunidades humanas; los derechos humanos contribuyen al desarrollo de las personas garantizando un espacio para que los grupos favorecidos no puedan monopolizar los procesos, las políticas y los programas de desarrollo. El marco de derechos humanos implica que los agentes institucionales tienen el deber de facilitar y fomentar el desarrollo para todas las personas sin discriminación por raza, sexo, nacionalidad u orientación sexual (Naciones Unidas, 2003); esta visión pretende superar el imperativo moral que establecía el enfoque basado en la satisfacción de las necesidades básicas de los años setenta para crear un marco de garantías para que la ciudadanía pueda reivindicar, de forma jurídicamente obligatoria, una serie de derechos (Naciones Unidas, 2003).

En tercer lugar, en tanto se trata de ampliar las opciones y oportunidades de toda la ciudadanía es un concepto que debe orientarse a promover la equidad en varios ámbitos: i) la promoción de la igualdad en la distribución de las opciones y oportunidades (económicas, sociales y políticas) de todas las personas; ii) la incorporación de la perspectiva de género adquiere una relevancia fundamental en tanto los derechos de las mujeres se han visto restringidos - aunque de manera divergente- en todas las sociedades humanas contemporáneas; iii) la asunción de que se trata de un concepto intrageneracional e intergeneracional en tanto la promoción de derechos de una generación no deben restringir los derechos de futuras generaciones. Por tanto, no serían políticas de desarrollo aquellas que promovieran o garantizaran las libertades de determinados grupos en detrimento de las opciones y libertades de otros ciudadanos, independientemente de la región o país a que pertenezcan.

A esta noción de desarrollo humano podrían integrarse otras concepciones filosóficas no occidentales como es el caso de concepto de Sumak Kawsay o el "Buen vivir" que apuesta por establecer un equilibrio entre la naturaleza y todos los seres vivos, destacando la importancia de la hermandad y espiritualidad en los países andinos (Cortez, 2009); el ecofeminismo, una corriente crítica con el orden simbólico patriarcal capitalista que se basa en un paradigma alternativo que sitúe en el centro la conservación de una vida humana (Herrero, 2012); y la economía del bien común que parte del fundamento de que la economía debe estar orientada a la 
promoción del bienestar social y el bien común cambiando el modelo productivo y ofreciendo a las empresas otro tipo de incentivos relacionados con la solidaridad, la justicia, el respeto a la naturaleza y la sostenibilidad (Felber, 2012).

En suma, el presente análisis parte de un concepto de desarrollo humano que supone la promoción de derechos de todas las personas - los cuales no deben ser restringidos por la pertenencia a un Estado o por cualquier otro rasgo en particular- y para ello, debe impulsarse una mayor equidad garantizando la mejora en el acceso a los derechos sociales, civiles, económicos, ambientales y políticos para todas las generaciones humanas. La Figura 1 pretende ilustrar los contenidos básicos del concepto de desarrollo humano propuesto en este artículo.

Figura 1. El concepto de Desarrollo Humano: implicaciones.

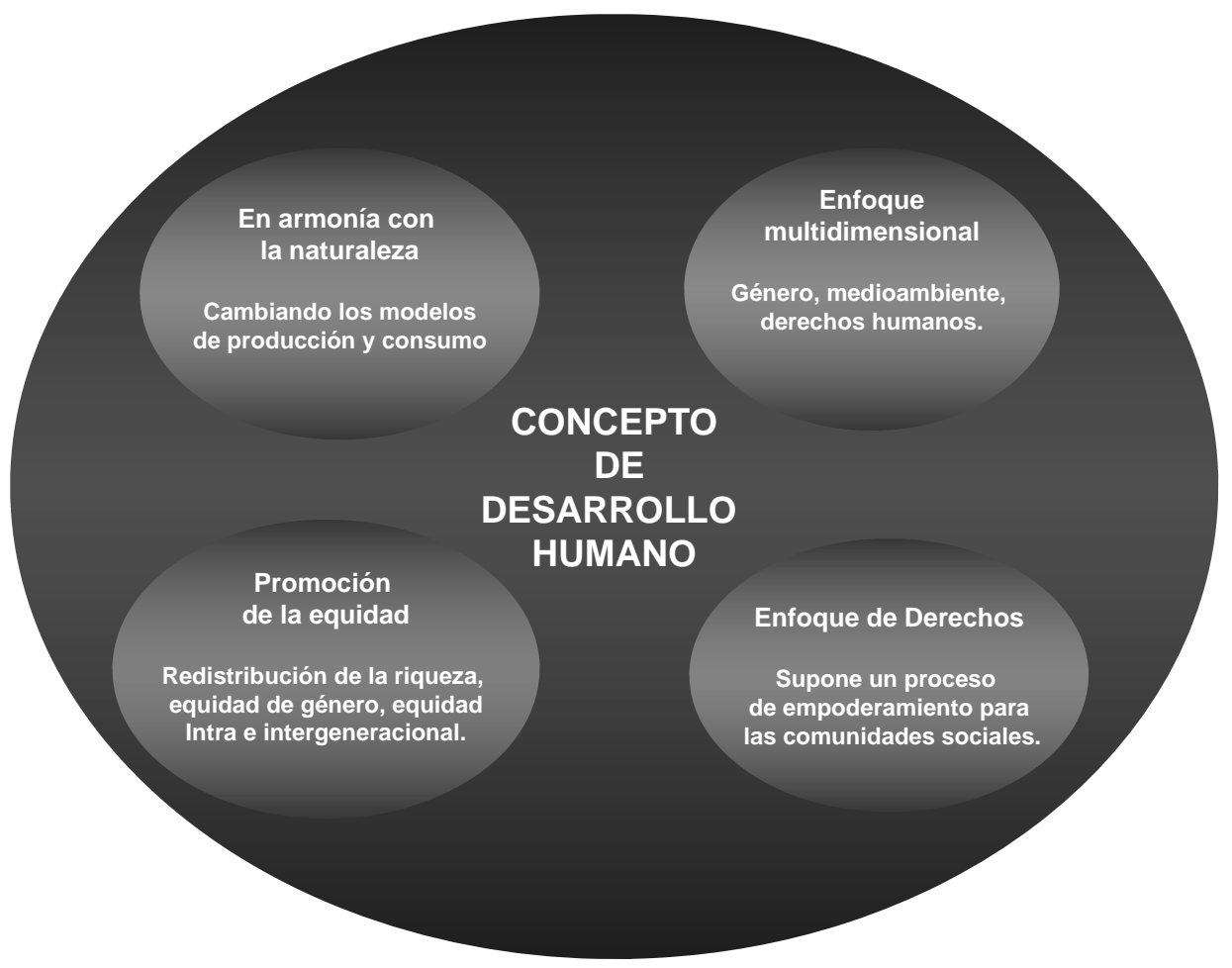

Fuente: elaboración propia. 


\section{El concepto de Coherencia de Políticas para el Desarrollo}

\subsection{Marco conceptual de la Coherencia de Políticas para el Desarrollo}

Existe un marco doctrinal relativamente amplio que, desde comienzos de los años noventa del siglo pasado, ha contribuido a la conceptualización del concepto de CPD. Si bien no hay una definición establecida de lo que ésta supone, existe un amplio consenso acerca de que la CPD implica la necesidad de ampliar la mirada hacia todas las políticas públicas más allá del análisis específico de la política cooperación; así, se pretende analizar el impacto global de las políticas públicas de un Estado, actor o institución en la promoción (o limitación) del desarrollo global y sustentable. Las diferentes definiciones que remiten a la CPD parten tanto de trabajos académicos como de instituciones internacionales y podrían categorizarse en tres tipos de aproximaciones teóricas.

En primer lugar, se encuentran aquellas definiciones que vinculan el concepto de coherencia a un ámbito de trabajo estrechamente vinculado con la política de cooperación. Así, la Coordinadora de ONG españolas entiende que la CPD debe perseguir "mejorar el impacto positivo de la cooperación asegurándose que las demás políticas están en consonancia con los objetivos de desarrollo y la lucha contra la pobreza" (Coordinadora, 2009:1). En la misma línea, CONCORD define a la coherencia como aquel conjunto de acciones que persiguen asegurar que los impactos externos de otras políticas de la UE no dañen los objetivos de la política de cooperación (CONCORD, 2009). Cabe destacar que se trata de concepciones que entienden la $\mathrm{CPD}$ en un sentido restrictivo o reactivo dado que consiste en "evitar" o "limitar" las inconsistencias o daños de otras políticas en el trabajo de la cooperación (Hoebink, 2003; Ashoff, 2005) aun cuando algunos autores consideran estas definiciones como el punto de partida sobre el cual empezar a trabajar en el ámbito de la coherencia (Barry et al., 2010). De todos modos, llama la atención que sean precisamente las organizaciones de la sociedad civil las que se establezcan una relación tan estrecha entre la agenda de la cooperación y la agenda de la CPD.

En segundo lugar, existen aproximaciones que pudieran considerarse más ambiciosas dado que abordan la promoción de sinergias o complementariedades entre políticas públicas. Aquí es posible ubicar a los autores que disciernen entre los conceptos de coherencia y consistencia ${ }^{2}$. Para Hoffmeister "la noción de consistencia se refiere a la ausencia de contradicciones mientras que la noción de coherencia se relaciona más con la promoción de sinergias positivas" (2008: 161) visión que

2 Dada la similitud de su significado en ingles diversos autores han señalado la diferencia entre coherencia y consistencia. Esta diferencia es fundamental en términos de medición dado que como se ha destacado por Missiroli, una política puede ser o no consistente mientras que por el contrario se puede medir la coherencia en diferentes grados (Missiroli, 2001). 
comparte los trabajos de Gauttier (2004) y Portela y Raube (2009) que identifican la coherencia con la promoción de sinergias entre políticas más que con evitar contradicciones entre ellas. El concepto de sinergia es fundamental en la visión de CPD que proponen Fukasku e Hirata al definirla como la necesaria congruencia entre los objetivos e instrumentos aplicados por los miembros de la OCDE y que puedan tener efectos sobre el progreso de los países del Sur (Fukasku, et al., 2005). En la misma línea Picciotto define la CPD como "la identificación de los intercambios y sinergias entre los ámbitos de la política hacia la consecución de los objetivos de desarrollo" (Picciotto, 2004:8). Para la UE "el concepto de coherencia de las políticas para el desarrollo tiene como objetivo crear sinergias entre las políticas y objetivos de desarrollo" (Unión Europea, 2007: 9). Una concepción más integral es la que han construido Foster y Stokke. Estos autores señalan que puede ser definida como coherente aquella política que: en primer lugar, presente objetivos internos formulados en forma clara y armonizada dentro de un marco dado las cuales deben ser consistentes con los objetivos perseguidos por otras políticas -o como mínimo no debieran ser contradictorios-; en segundo lugar, donde las estrategias y los instrumentos se encuentren en sintonía con los objetivos planteados -o como mínimo no debieran ser conflictivos con estos objetivos o con los compromisos y las intenciones asumidas por el resto de las políticas gubernamentales-; y en tercer lugar, donde los resultados se corresponden a las intenciones y objetivos -o como mínimo no entran en conflicto con estos - (Forster y Stokke, 1999). Por último, cabe destacar la aproximación a la CPD que propone la OCDE en su último informe sobre esta temática; en este se explica que al CPD es un concepto multidimensional y complejo que supone tanto la identificación de contradicciones como la promoción de sinergias entre políticas públicas (OCDE, 2014).

En el último nivel, se han elaborado conceptualizaciones teóricas que pueden considerarse más complejas e integrales para abordar el concepto de coherencia: son las que suponen transversalizar el enfoque de desarrollo en toda la acción gubernamental más allá de evitar posibles contradicciones o promover sinergias entre las políticas públicas (Ashoff, 2005; Barry et al., 2010). A esta perspectiva, algunos donantes han llamado the whole of goverment approach. En teoría, asumir este "enfoque integral orientado al desarrollo" supondría que los países deberían considerar la perspectiva de desarrollo como un área prioritaria (aunque no necesariamente única) en los procesos de toma de decisiones públicas. Así lo han concebido algunos donantes del CAD entre los que se puede mencionar a Suecia que posee, desde 2003, una Política Global de Desarrollo que involucra a todos los ministerios y entes gubernamentales (Gobierno de Suecia, 2008). Esta visión supone una responsabilidad compartida que debe ser asumida por todas las instancias de la administración pública más allá de la agenda específica de la política de cooperación. Dentro de este contexto, la CPD supone tomar en consideración las necesidades e intereses de los países en desarrollo a la hora de diseñar las políticas ajenas a la AOD (Carbone, 2009). 
Figura 2. Distintas visiones de la CPD.

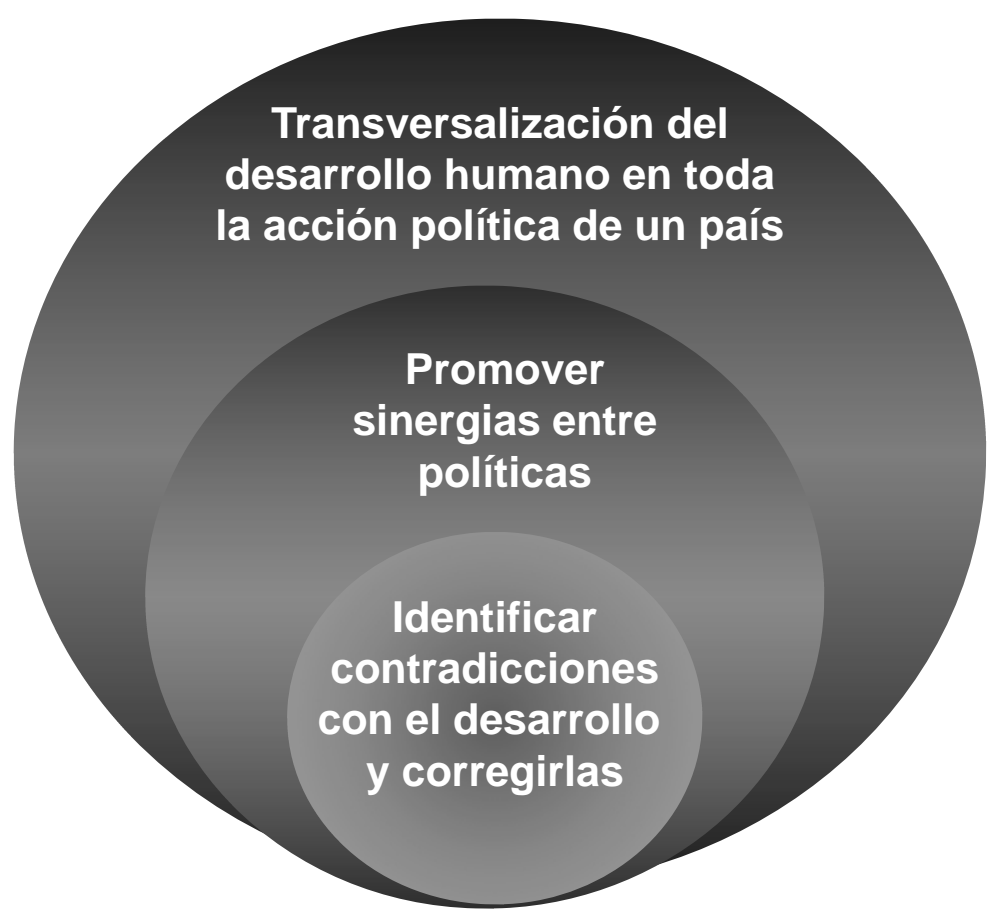

Fuente: elaboración propia.

\subsection{Conceptualización de la CPD}

Teniendo en cuenta lo expuesto hasta el momento, el presente artículo conceptualiza al trabajo por la CPD como la integración de la perspectiva de desarrollo en el diseño, implementación y evaluación de todas las políticas públicas de un país, lo que significa transversalizar el trabajo por el desarrollo en toda la acción gubernamental (Millán, 2012). Asimismo, los fenómenos de interdependencia han reconfigurado los procesos de desarrollo, convirtiendo la pobreza y la desigualdad en desafíos globales que afectan a todos los países en su conjunto. Desde esta perspectiva, la CPD también se convierte en un imperativo en la acción cooperativa entre Estados, lo que obliga a avanzar hacia soluciones coordinadas y coherentes con el desarrollo, las cuales permitirían promover la construcción de un mundo más equitativo y estable para todo el sistema internacional (Millán, 2012).

El propósito de la CPD supone, en principio, un compromiso integral y global del gobierno en la promoción del desarrollo, aunque no deja, sin embargo, de constituirse como un propósito extremadamente ambicioso y con importantes problemas 
de implementación (Alonso et al., 2010). Se trata, pues, de un concepto sugerente, puesto que el fin último del trabajo por la coherencia consiste en modificar progresivamente las políticas públicas para integrar de manera transversal la perspectiva de desarrollo humano. En tanto las fronteras entre las dimensiones domésticas e internacionales son cada vez más difusas y porosas, la agenda adquiere una dimensión "interméstica" (Evans et al., 1993) por lo que la visión del desarrollo humano deberá transversalizar tanto las políticas domésticas como internacionales, asumiendo que todas ellas poseen importantes consecuencias en las posibilidades de progreso de otros países y personas.

Esta visión de CPD descansa sobre cuatro supuestos:

En primer lugar, se trata de una visión cosmopolita del concepto de CPD. De acuerdo a esta doctrina, la ciudadanía no puede fundarse en la nacionalidad como vínculo de inclusión/exclusión a una comunidad política sino que, en la medida en que se basa en la titularidad de unos derechos humanos inalienables ejercidos en el espacio público, los derechos ciudadanos deben extenderse a todos aquellos que comparten dicho espacio que, en su límite, coincide con el mundo entero. El concepto de CPD desde una perspectiva cosmopolita supone trascender y superar la dicotomía global/local que se utiliza de manera sistemática tanto para separar los intereses de las naciones como para dividir y compartimentalizar los derechos ciudadanos. Así, en diversas ocasiones se utiliza un recurso discursivo que parece concebirse al Estado como un ente monolítico que únicamente "compite" con los intereses de otros Estados. Las estrategias más recientes de "marca país" parecen ilustrar de manera meridiana la visión la competitividad como indicador máximo de la evolución de un país (y de su ciudadanía) (Millán, 2013).

Así, en esta nueva realidad globalizada se han desarrollado redes económicas y financieras que favorecen a actores que ya no se encuentran "contenidos y delimitados" por el territorio de un Estado en particular (Beck, 2005; Held, 2004). En este sentido, más que una contraposición entre los derechos de unos ciudadanos y otros, las políticas públicas pueden favorecer o perjudicar a grupos de intereses y actores que trascienden las fronteras de los Estados. Por tanto, es necesario trascender la dicotomía entre ciudadanos del "Sur" o del "Norte" para comprehender que el trabajo por el desarrollo humano se orienta a promover los derechos de la ciudadanía cosmopolita.

En segundo lugar, se establece que la acción de un Gobierno tiene que orientarse a la promoción del desarrollo humano. Cabe recordar que el concepto de Coherencia posee un "valor instrumental" que se subordina a los objetivos que se definen como prioritarios en el seno de una administración pública (Alonso, 2010). Es decir, la consistencia entre objetivos, valores, políticas e instrumentos puede estar condicionada por diversos intereses, entre los cuales el desarrollo humano no es necesariamente una prioridad. Cabe recordar, que a pesar de que entre los responsables gubernamentales parece existir una conciencia clara de que es necesario avanzar hacia una mayor coherencia de políticas públicas (desde una perspectiva más bien técnica), el verdadero desafío es determinar (y consensuar) cuál es el fin último que debe perseguir esta coherencia. El priorizar al desarrollo humano como elemento 
básico de la CPD supone una opción política en la que se asume que el fin último de la acción pública debe ser la promoción de los derechos humanos y el bienestar de las personas.

En este sentido, el "enfoque integral del Gobierno" mencionado anteriormente - que implica avanzar hacia acciones más coherentes y concertadas - no siempre se ha utilizado para promover agendas vinculadas a la promoción del desarrollo global. Así, en diversas ocasiones los gobiernos esgrimen el argumento de la coherencia de políticas como coartada para supeditar diversas programas a la promoción de intereses nacionales bajo el paradigma de la realpolitik (Sanahuja y Schüneman, 2012). Un ejemplo ilustrativo son las políticas de seguridad desarrolladas por algunos donantes, (principalmente EEUU) que siguiendo la narrativa de la "Guerra Global contra el Terror", han utilizado la ayuda en un marco fuertemente securitizado que responde a objetivos internos y externos de seguridad del Estado y que ha reorientado la cooperación al desarrollo y la ayuda humanitaria en función de necesidades estratégicas de seguridad En tal sentido, cabe destacar que la coherencia debe ser entendida como la promoción de objetivos de desarrollo y no de agendas "nacionales" impulsadas por los intereses cortoplacistas de los diferentes Estados donantes.

En tercer lugar, si bien en un sistema democrático coexisten por definición intereses contradictorios y legítimos, el trabajo por el desarrollo humano deberá ser tenido en cuenta en todo el ciclo de acción política. En este sentido, los países representan delicadas y complejas combinaciones de intereses, grupos, estándares y normas. Alcanzar un grado absoluto de coherencia puede convertirse, por tanto, en un objetivo incompatible y poco deseable para un sistema plural, abierto y participativo (Alonso, 2003). No obstante, el trabajo por la CPD consiste en que la perspectiva de desarrollo humano debe estar presente en los procesos de toma de decisiones, aun cuando este enfoque no sea la única o la principal prioridad de una política en particular.

Por último, dentro del contexto de globalización explicado, la agenda de la CPD no debería restringirse a una responsabilidad exclusiva de los países desarrollados; en este sentido, los procesos de interdependencia mencionados exigen que todos los Estados entiendan que las políticas que ponen en práctica tienen una importante incidencia en las posibilidades de desarrollo de otros países y personas. En este sentido, la integración del concepto de CPD no sólo supondría mejorar las posibilidades de desarrollo global sino también que los países (independientemente de su estadio de desarrollo) asuman la responsabilidad de considerar las consecuencias de su acción pública para otras sociedades y personas.

Incorporando los elementos analizados hasta el momento así como la visión del desarrollo que se ha propuesto en epígrafes anteriores varias son las implicaciones que contiene el concepto de CPD como se ilustra en la figura 3. 
Figura 3. El concepto de Coherencia de Políticas para el Desarrollo y sus implicaciones.

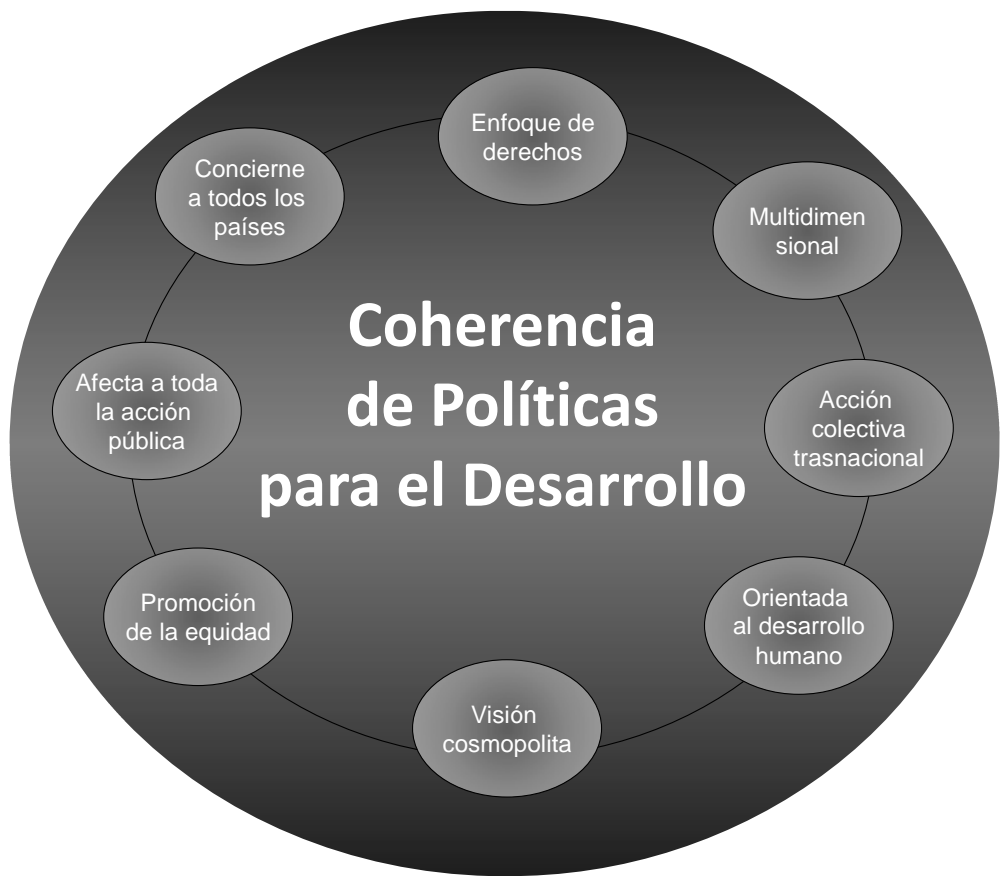

Fuente: elaboración propia.

\section{Una propuesta teórica metodológica para un análisis comprehensivo de la CPD}

El objetivo de este epígrafe, es desarrollar una propuesta metodológica - de tipo cualitativa - comprehensiva para estudiar la CPD basado en cuatro dimensiones analíticas. Para ello, se propone utilizar tres dimensiones ya elaboradas por diversos estudios académicos - aunque como se verá, estas dimensiones se estructuran y profundizan en trabajo-, así como una nueva dimensión cuyo estudio permitiría completar un enfoque integral de análisis de CPD. Con este propósito, se detallan en primer lugar, las tres dimensiones ya propuestas y la estructura de análisis que debiera seguirse para desarrollar el enfoque propuesto sobre CPD.

La primera dimensión alude a lo que se entiende como coherencia vertical o interna de una política propuesta por la OCDE (2000); la coherencia vertical remite a la convergencia entre valores, compromisos, acciones y sistemas de una política en particular (Millán, 2011). Aunque este concepto se encuentra estrechamente vinculado a la eficacia (Alonso et al., 2010) también se refiere a la necesaria convergencia e integración de actores y agendas que forman parte de una política pública. Por 
ende, la coherencia vertical garantiza que todas las instancias de diseño, implementación y evaluación respondan a los compromisos y objetivos que una política presume perseguir, aunque es fundamental destacar que estos elementos deben analizarse en función de la visión de desarrollo humano que se haya asumido. Esta visión puede referir a cualquier ámbito de la acción pública por lo que es posible analizar la coherencia vertical en cualquier marco de políticas como puede ser la política comercial, migratoria, de defensa o medioambiental (Stokke, 2003).

Asimismo, esta dimensión de la coherencia también pudiera aplicarse a otros ámbitos que no remiten únicamente a las acciones públicas como es la coherencia interna en la actuación de otros actores de desarrollo, como pueden ser las empresas o las Organizaciones No Gubernamentales de Desarrollo (ONGD). En tal sentido, la convergencia entre los compromisos, los discursos, la comunicación pública y las acciones de estos actores es un ámbito que es preciso empezar a analizar bajo la óptica de la CPD, aunque hasta el momento no se trata de una práctica desarrollada por los estudios académicos sobre el tema.

Cabe recordar, que la coherencia vertical trata de analizar una política en particular y su convergencia o divergencia con la promoción del desarrollo humano. Es decir, el objetivo de un programa de investigación de coherencia vertical es valorar si esta política integra la visión del desarrollo humano, y cuáles podrían ser las implicaciones de dicha política para los derechos humanos de la ciudadanía afectada por la misma. En este sentido, cabe incorporar a esta dimensión una esfera que se suele omitir en los análisis de CPD que debería aludir a la dimensión doméstica de la política valorada. Así, es necesario estudiar no sólo las implicaciones de esta política pública para otros países y personas sino también para la propia población que vive en este Estado. Si como se ha explicado, el trabajo por la CPD debería orientarse a la ampliación de las libertades y opciones de los ciudadanos (independientemente de su nacionalidad), un ámbito principal son las consecuencias de una política para los derechos de su ciudadanía que vive en este país.

Por último, cabe incorporar otro ámbito de especial relevancia en la CPD, aunque se trata de una esfera poco valorada en los estudios académicos sobre CPD. Se trata de la posible capacidad de interferencia de actores y lobbies privados en el diseño de políticas públicas (Millán y Santander, 2014).

El Cuadro 1 resume las principales preguntas de investigación que deben guiar el análisis de CPD vertical. 
Cuadro 1. Preguntas de investigación guía para la dimensión vertical de la CPD.

\begin{tabular}{|c|l|}
\hline \multicolumn{2}{|c|}{ Dimensión vertical de la CPD. Preguntas de investigación. } \\
\hline 1 & $\begin{array}{l}\text { ¿Cuáles son los objetivos que esta política persigue y cuáles son las relaciones de } \\
\text { estos objetivos con la promoción del desarrollo humano? }\end{array}$ \\
\hline 2 & $\begin{array}{l}\text { ¿En el ámbito discursivo /doctrinal, integra esta política un compromiso concreto } \\
\text { con la perspectiva de desarrollo humano? }\end{array}$ \\
\hline 3 & $\begin{array}{l}\text { ¿Existe un marco legal específico que obligue a incorporar la visión del desarrollo } \\
\text { humano al diseño de esta política? }\end{array}$ \\
\hline 4 & $\begin{array}{l}\text { ¿Concurren mecanismos que permitan integrar la perspectiva de desarrollo a la } \\
\text { implementación de esta política? }\end{array}$ \\
\hline 5 & $\begin{array}{l}\text { ¿Cuáles son los intereses, valores y expectativas de los actores directamente } \\
\text { implicados en el diseño y ejecución de esta política? }\end{array}$ \\
\hline 6 & $\begin{array}{l}\text { ¿Se han establecido mecanismos ex ante y ex post que permitan evaluar las } \\
\text { implicaciones de esta política para el desarrollo humano? }\end{array}$ \\
\hline 7 & $\begin{array}{l}\text { ¿Existen interferencias externas (actores privados) en el diseño de esta política? y } \\
\text { ¿Se trata de interferencias que pueden afectar las implicaciones de la misma para } \\
\text { el desarrollo humano? }\end{array}$ \\
\hline 8 & $\begin{array}{l}\text { ¿Cuáles son las implicaciones de las políticas públicas para las posibilidades de } \\
\text { desarrollo de otros países? }\end{array}$ \\
\hline 9 & $\begin{array}{l}\text { ¿Cuáles podrían ser las consecuencias de estas políticas para los derechos huma- } \\
\text { nos de la ciudadanía cosmopolita (nacional e internacional)? }\end{array}$ \\
\hline
\end{tabular}

Fuente: elaboración propia.

En segundo lugar, y siguiendo a la OCDE (2000), la coherencia horizontal alude a la convergencia entre diferentes políticas públicas. En esta dimensión se analiza si la las estructuras, actores, mecanismos y procesos de un Gobierno tiende a promover el desarrollo humano o, en sentido negativo, si estas estructuras omiten la visión del desarrollo y, por tanto, tendrán consecuencias negativas para la ciudadanía cosmopolita. Esta dimensión de la coherencia alude a la acción global del gobierno, lo que muchos donantes y teóricos han denominado "the whole of government approach" (Alonso et al., 2010). En esta dimensión, también se encuadra el concepto de coherencia como sistema (Millán, 2010); así para avanzar en una meta tan compleja como la CPD es necesario el diseño de un sistema de instrumentos relacionados que afecten a la diversa variedad de políticas e instrumentos gubernamentales. Un enfoque realmente integral no puede alcanzarse a través de instrumentos aislados o puntuales y cada sistema deberá estar adaptado a las capacidades, estructuras administrativas y culturas institucionales que caracterizan a los diferentes donantes; como en todo ámbito de desarrollo no existe, por tanto, una receta universal idónea para promover la CPD (OCDE, 2008).

El objetivo de un programa de estudio de CPD horizontal debiera ser analizar los factores transversales que afectan a la acción pública de un Gobierno, en función, 
del desarrollo humano. Cabe destacar que el estudio de los mecanismos, actores y procesos se encuentran contextualizados en un entorno organizacional socialmente construido que rige, estructura, premia y castiga la acción política. Por tanto, es fundamental en este punto el valorar los factores "ideacionales" (Sanahuja, 2007) que afectan al diseño de políticas, es decir, los intereses, valores e identidades que guían a los decidores públicos y que son ineludibles para comprender el posible avance o retroceso en CPD.

El siguiente Cuadro resume las principales preguntas de investigación de la CPD en su dimensión horizontal.

Cuadro 2. Preguntas de investigación guía para la dimensión horizontal de la CPD.

\begin{tabular}{|c|l|}
\hline \multicolumn{2}{|c|}{ Dimensión horizontal de la CPD. Preguntas de investigación. } \\
\hline 1 & $\begin{array}{l}\text { Existe un compromiso integral al más alto nivel jerárquico con la CPD? Y si es así, } \\
\text { ¿Cuál es la visión doctrinal que se tiene de CPD en el marco gubernamental? }\end{array}$ \\
\hline 2 & $\begin{array}{l}\text { ¿Existe un marco legal que garantice el cumplimiento de la CPD en la acción pública } \\
\text { del Gobierno? }\end{array}$ \\
\hline 3 & $\begin{array}{l}\text { ¿Se han establecido mecanismos formales que permiten que la visión del desarrollo } \\
\text { humano se incorpore en el diseño de políticas públicas? ¿Cuál es el diseño institucio- } \\
\text { nal de estos mecanismos? ¿Se adapta éste a las características particulares del país? }\end{array}$ \\
\hline 4 & ¿Cómo afecta la cultura organizacional del país a la promoción de la CPD? \\
\hline 5 & $\begin{array}{l}\text { ¿Cuáles son las percepciones de los actores estatales con respecto al trabajo para el } \\
\text { desarrollo humano? ¿Es la agenda del desarrollo entendida como un ámbito priorita- } \\
\text { rio en la acción pública del Gobierno? }\end{array}$ \\
\hline 6 & $\begin{array}{l}\text { ¿Concurren actores institucionales (equipos especializados) que trabajen para promo- } \\
\text { ver y asegurar la integración del desarrollo humano en el diseño e implementación de } \\
\text { políticas públicas? }\end{array}$ \\
\hline 7 & $\begin{array}{l}\text { ¿Se han elaborado mecanismos de seguimiento y evaluación que permitan valorar la } \\
\text { integración de la CPD en las políticas públicas? }\end{array}$ \\
\hline 8 & $\begin{array}{l}\text { ¿Existen mecanismos de transparencia y rendición de cuentas en cuanto al impacto de } \\
\text { estas políticas para otros países y personas? }\end{array}$ \\
\hline
\end{tabular}

Fuente: elaboración propia.

En tercer lugar, se alude a la dimensión temporal de la coherencia (OCDE, 2000) que se relaciona con la consistencia que a largo plazo debe asumir una política pública si pretende alcanzar verdaderos resultados de desarrollo. Es necesario destacar que el avance hacia una mayor coherencia es una tarea compleja que requiere un compromiso de largo plazo que exceda el ciclo político de un gobierno específico (ECDPM e ICEI, 2006). Por esto, el consenso entre todo el espectro político acerca de la importancia del desarrollo debe considerarse como un requisito fundamental para avanzar en este campo (Millán, 2011), este objetivo parece complejo si se considera la dinámica acelerada de transformaciones que se suceden en la actual etapa globalizadora y que hace más dificultoso, si cabe, que los responsables 
políticos asuman compromisos a largo plazo (y que los cumplan). La reciente crisis económica que ha afectado a gran parte de los países desarrollados parece dar cuenta de las dificultades, interferencias y presiones que se ejercen sobre los gobernantes y que limitan los espacios de toma de decisiones nacionales.

Además, para que la CPD pueda mantenerse como una prioridad a largo plazo, es necesario contar con una opinión pública firme y comprometida con los problemas del desarrollo global. En consecuencia, la educación formal e informal parece un elemento cardinal para avanzar en el trabajo por el desarrollo humano. Asimismo, el trabajo de las ONGD y de los movimientos sociales es indispensable para movilizar y sensibilizar a la población. Por último, un ámbito poco analizado en los estudios de CPD, pero que debe ser valorado es el papel de los medios de comunicación en la formación de la opinión pública.

En definitiva, un programa de estudio de la coherencia temporal remite a dos ámbitos que se encuentran interrelacionados; en primer lugar, el interés de la ciudadanía en la promoción del desarrollo humano y el trabajo de incidencia de los actores de la sociedad civil; y, en segundo lugar, el compromiso político sostenido por parte de los gestores y responsables políticos. En este marco, las preguntas de investigación que deberían guiar un análisis de CPD temporal son las siguientes:

Cuadro 3. Preguntas de investigación guía para la dimensión temporal de la CPD.

\begin{tabular}{|c|l|}
\hline \multicolumn{2}{|c|}{ Dimensión temporal de la CPD. Preguntas de investigación. } \\
\hline 1 & $\begin{array}{l}\text { Existe un compromiso político entre todos los partidos, acerca de la importancia de } \\
\text { integrar la visión del desarrollo humano a la acción pública estatal? }\end{array}$ \\
\hline 2 & $\begin{array}{l}\text { ¿Se han desarrollado programas de formación entre funcionarios/as y responsables } \\
\text { políticos acerca de la importancia de integrar la CPD a la agenda política? }\end{array}$ \\
\hline 3 & $\begin{array}{l}\text { ¿Se solicita formación y/o conocimiento en temas de desarrollo y derechos humanos } \\
\text { en las oposiciones para cargos públicos? }\end{array}$ \\
\hline 4 & $\begin{array}{l}\text { ¿Se encuentra el trabajo por el desarrollo y los derechos humanos presentes en los } \\
\text { programas de los partidos políticos? }\end{array}$ \\
\hline 5 & ¿Los problemas del desarrollo humano son parte del debate político? \\
\hline 6 & $\begin{array}{l}\text { ¿Existen comisiones parlamentarias orientadas al análisis de los temas de desarrollo } \\
\text { humano? }\end{array}$ \\
\hline 7 & $\begin{array}{l}\text { ¿La enseñanza pública, integra la visión del desarrollo humano en todas sus áreas de } \\
\text { manera de crear una opinión pública comprometida? }\end{array}$ \\
\hline 8 & $\begin{array}{l}\text { ¿Existe una sociedad civil organizada que apuesta por el trabajo de incidencia política } \\
\text { y educación para el desarrollo? }\end{array}$ \\
\hline 9 & ¿Existe espacio en los medios para los temas de desarrollo y derechos humanos? \\
\hline 10 & $\begin{array}{l}\text { ¿Se han establecido sistemas de seguimiento de la actividad parlamentaria en estos } \\
\text { ámbitos? }\end{array}$ \\
\hline
\end{tabular}

Fuente: elaboración propia. 
Hasta aquí, el análisis propuesto se orienta a valorar las políticas públicas en relación con la CPD en tres aspectos: i) en función de la integración de la perspectiva de desarrollo en el diseño e implementación de una política pública (vertical); ii) en función de la agenda que ocupa la CPD en la agenda gubernamental y los mecanismos, actores y procesos que influyen en esta agenda (horizontal); y, iii) en función de los factores que promuevan la convergencias o divergencia de la agenda del desarrollo en el tiempo (temporal).

Ahora bien, más allá de las dimensiones mencionadas, y teniendo en cuenta los procesos de interdependencia e interrelación derivados de la dinámica de la globalización, la coherencia de políticas debería integrar también una "dimensión cosmopolita o trasnacional" orientada a promover la acción colectiva, concertada y democrática entre todos los países. Debido a que la problemática del desarrollo es un fenómeno trasnacional que no puede ni debe abordarse desde respuestas nacionales y segmentadas no basta (únicamente) con impulsar políticas nacionales coherentes sino que es necesario promover una acción colectiva y global entre los diversos Estados del planeta. En este sentido, la dimensión trasnacional que alude a la necesaria acción colectiva y coordinada orientada al desarrollo parece ser el elemento fundamental para afrontar desafíos y amenazas que escapan al control de los Estados y cuestionan la sustentabilidad del sistema en su conjunto.

En este marco, es necesario desarrollar una agenda de investigación orientada a analizar las acciones que emprenden los Estados para promover una acción cooperativa con otros Estados orientados a gestionar colectivamente problemas que se han transnacionalizado. Así, las posiciones y acciones que se adoptan en los organismos multilaterales y los mecanismos orientados a promover una acción concertada son fundamentales en esta agenda de investigación. Además, es necesario valorar si existe un espacio analítico y doctrinal para avanzar en la agenda de la CPD. Un caso particular de análisis en esta dimensión es la Unión Europea en tanto sus Estados Miembros poseen competencias atribuidas a las instituciones comunitarias. En ese caso, será necesario también analizar la CPD en función de las decisiones y acciones que toma la UE, dado que los Estados miembros son co-responsables de las mismas. Por tanto, cuando se realiza un análisis del resultado de la (in)coherencia en las políticas para el desarrollo con respecto a un país que forma parte de la Unión Europea, se debe recordar que se trata de un ámbito que remite tanto a la posición del Estado miembro como a las decisiones finales que se toman en su seno. Por último, es necesario valorar los mecanismos e instancias de diálogo con los países en desarrollo, dado que gran parte de las políticas públicas de los países desarrollados pudieran afectar a los países más pobres.

En este marco, las preguntas de investigación que podrían guiar una agenda de investigación de CPD trasnacional serían las siguientes: 
Cuadro 4. Preguntas de investigación guía para la trasnacional o cosmopolita de la CPD.

\begin{tabular}{|l|l|}
\hline \multicolumn{2}{|c|}{ Dimensión cosmopolita o trasnacional de la CPD. Preguntas de investigación. } \\
\hline 1 & $\begin{array}{l}\text { ¿Existe espacio político y discursivo para una doctrina cosmopolita que incentive al } \\
\text { Estado a promover acciones colectivas con otros países y actores? }\end{array}$ \\
\hline 2 & $\begin{array}{l}\text { ¿Es consciente el poder político (partidos y oposición) de la importancia de la transna- } \\
\text { cionalización del desarrollo y la necesidad de generar acciones cooperativas entre } \\
\text { Estados? }\end{array}$ \\
\hline 3 & $\begin{array}{l}\text { ¿Participan los Estados de forma activa en organismos multilaterales de acción coopera- } \\
\text { tiva de gestión de males y bienes públicos globales? }\end{array}$ \\
\hline 4 & $\begin{array}{l}\text { ¿Cuáles son los tratados y convenios internacionales (relacionados con el desarrollo } \\
\text { humano) que los Estados han firmado y ratificado? }\end{array}$ \\
\hline 5 & $\begin{array}{l}\text { ¿Cuáles son las posiciones que el Estado asume con respecto al desarrollo humano en } \\
\text { los distintos foros internacionales? }\end{array}$ \\
\hline 6 & $\begin{array}{l}\text { ¿Existen mecanismos de coordinación con otros actores bilaterales para trabajar en } \\
\text { conjunto los problemas del desarrollo? }\end{array}$ \\
\hline 7 & $\begin{array}{l}\text { ¿Existen instancias de diálogo con otros países que pudieran ser afectados por las } \\
\text { políticas desarrolladas? }\end{array}$ \\
\hline
\end{tabular}

Fuente: elaboración propia.

En definitiva, se propone un programa de investigación en CPD que pretende ser una guía para orientar y clarificar los estudios en esta materia. Para ello, se proponen cuatro dimensiones que atienden a diversas esferas de la CPD pero que se encuentran interrelacionadas entre sí. El propósito último de este ejercicio, es desarrollar un marco metodológico que permita un estudio comprehensivo y acabado sobre la CPD. 
Cuadro 5. Las cuatro dimensiones de la CPD.

\begin{tabular}{|c|c|c|c|c|}
\hline Dimensiones & $\begin{array}{l}\text { Coherencia } \\
\text { cosmopolita }\end{array}$ & $\begin{array}{l}\text { Coherencia } \\
\text { vertical }\end{array}$ & $\begin{array}{l}\text { Coherencia } \\
\text { horizontal }\end{array}$ & $\begin{array}{c}\text { Coherencia } \\
\text { temporal }\end{array}$ \\
\hline $\begin{array}{c}\text { Ámbitos a los } \\
\text { que se } \\
\text { remiten }\end{array}$ & $\begin{array}{l}\text { Avance hacia una } \\
\text { acción colectiva } \\
\text { entre países e } \\
\text { instituciones } \\
\text { internacionales }\end{array}$ & $\begin{array}{c}\text { Una política } \\
\text { pública específica } \\
\text { en relación con el } \\
\text { desarrollo hu- } \\
\text { mano. }\end{array}$ & $\begin{array}{l}\text { Mecanismos, actores } \\
\text { y procesos en la } \\
\text { promoción trasnver- } \\
\text { sal del desarrollo } \\
\text { humano. }\end{array}$ & $\begin{array}{l}\text { Consistencia en el } \\
\text { tiempo con el } \\
\text { desarrollo } \\
\text { humano. }\end{array}$ \\
\hline \multirow{4}{*}{$\begin{array}{c}\text { Actores } \\
\text { involucrados }\end{array}$} & $\begin{array}{c}\text { Gobiernos donantes } \\
\text { y socios }\end{array}$ & $\begin{array}{l}\text { Gobierno, directi- } \\
\text { vos y funcionarios } \\
\text { públicos involu- } \\
\text { crados en el diseño } \\
\text { de la política a } \\
\text { estudiar }\end{array}$ & $\begin{array}{l}\text { Presidencia y minis- } \\
\text { terios }\end{array}$ & Partidos políticos \\
\hline & \multirow{3}{*}{$\begin{array}{l}\text { Organismos y foros } \\
\text { multilaterales }\end{array}$} & $\begin{array}{c}\text { Actores y lobbies } \\
\text { privados }\end{array}$ & $\begin{array}{c}\text { Entes gubernamenta- } \\
\text { les }\end{array}$ & Opinión pública \\
\hline & & \multirow[b]{2}{*}{$\begin{array}{l}\text { Actores de la } \\
\text { sociedad civil }\end{array}$} & \multirow[b]{2}{*}{$\begin{array}{l}\text { Actores de la socie- } \\
\text { dad civil }\end{array}$} & Parlamento \\
\hline & & & & $\begin{array}{c}\text { Organizaciones } \\
\text { de la Sociedad } \\
\text { Civil }\end{array}$ \\
\hline
\end{tabular}

Fuente: elaboración propia.

\section{Reflexiones finales}

La agenda de la CPD parece ser cada vez más necesaria en un mundo crecientemente interdependiente donde los problemas del desarrollo se han transnacionalizado, $\mathrm{y}$, por ende, las posibilidades de progreso de los países y personas depende de las interrelaciones con otros países y de las oportunidades y limitaciones que ofrezca el escenario internacional.

Sin embargo, aún no parece haber un consenso sobre el concepto de CPD así como el enfoque que debe asumir un programa de investigación sobre este tema. En este marco, dos son los elementos principales que han orientado este trabajo. En primer lugar, desarrollar una definición comprehensiva sobre lo que es la CPD, su objeto de estudio y los supuestos e implicaciones sobre los se asienta este concepto. En segundo lugar, establecer una propuesta de marco metodológico comprehensivo para analizar la CPD.

Cabe destacar, en este aspecto, que los estudios académicos sobre CPD, no sólo parten de una visión distinta sobre lo que es este concepto sino con enfoques diversos sobre cómo abordar dicho objeto de estudio. Por ello, se ha propuesto un marco de estudio basado en cuatro dimensiones que pretenden ser una guía que permita analizar de manera comprehensiva las oportunidades y desafíos en que concurren 
los Gobiernos en la promoción de la CPD. Estas dimensiones, remiten tanto a la contribución de la acción pública de un Gobierno a la promoción del desarrollo humano como a los esfuerzos para impulsar una acción colectiva que permitan gestionar a escala global los nuevos desafíos del desarrollo que se han vuelto trasnacionales. Se trata de una propuesta basada en la metodología del estudio de caso, de corte cualitativo y que pretende asumir un enfoque multidimensional para estudiar un objeto complejo como es la CPD.

Teniendo en cuenta que el trabajo por la CPD consiste en la integración paulatina del mandato de desarrollo al diseño de políticas públicas, la utilidad de una propuesta metodológica es doble. El objeto del artículo es, por tanto, contribuir a la mejora del conocimiento sobre la CPD y a los estudios que se elaboren sobre este tema. Cabe destacar, en este sentido, que un objetivo fundamental del estudio y la práctica de las ciencias sociales es desarrollar un conocimiento pragmático que brinde herramientas teóricas para mejorar las estructuras sociales y las opciones de desarrollo de las personas que viven en este mundo. Y este es precisamente el objeto de la agenda de la Coherencia de Políticas para el Desarrollo.

\section{Bibliografía}

Alonso, J. A., P. Aguirre, R. Madrueño y N. Millán (2010): Coherencia de políticas para el desarrollo en cinco donantes del CAD: lecciones para el caso español, Documento de trabajo número 42, Madrid, Publicaciones Fundación Carolina.

Ashoff, G. (2005): "Improving Policy Coherence for Development: Conceptual Issues, Institutional Approaches and Lessons from Comparative Evidence in the EU", en Insecurity and Development: Regional Issues and Policies for an Interdependent World, Bonn, German Development Institute.

Barry, F. M. King y A. Matthews, (2010): "Policy Coherence for Development: Five Challenges", Irish Studies in International Affairs, 21, pp. 207-223.

Beck, U. (2005): La mirada cosmopolita o la guerra es la paz, Barcelona, Paidós.

Carbone, M. (2009): "Mission Impossible: The European Union and Policy Coherence for Development", Journal of European Integration, pp. 323-342.

Coordinadora. (2009): La coherencia de políticas para el desarrollo. Urge Otra Europa, Disponible en web: http://www.urgeotraeuropa.org/mm /file/Ficha\%20CPD.pdf. Fecha de consulta: 17/08/2011.

Cortez, D. (2009): "La construcción social del "Buen Vivir" (Sumak Kawsay), en Ecuador, Genealogía del diseño y gestión política de la vida, Actas del Congress for Intercultural Philosophy: Good life as Humanized life, v. VIII, Seúl, Ewha Womans University, pp 5-10,

ECDPM e ICEI. (2007): Evaluation study on the EU institutions and member state mechanism for promoting policy coherence for development, Amsterdam, Askant Academic Publishers

Evans, P., H. Jacobson y R. Putnam (1993): Double-Edge Diplomacy. International Bargaining and Domestic Politics, Berkeley, University of California Press. 
Foster, J. y O. Stokke, (1999): Policy Coherence in Development Cooperation, Londres, Frank Class Publishers.

Fukasaku, K., M. Plummer y J. Tan (1995): OECD and the ASEAN Economies: The Challenge of Policy Coherence, Development Centre Documents, Paris, OECD Development Centre.

Gauttier, P. (2004): "Horizontal Coherence and the External Competencies of the European Union", European Law Journal, 10, pp. 23-41.

Gobierno de Suecia (2008): Global Challenges. Our Responsibilities, Estocolmo, MAE.

Held, D. (2004): Un pacto global, Madrid, Taurus.

Hoebink, P. (2003): "La lucha por el equilibrio: coherencia y política para el desarrollo", en Alonso, J.A. y Fitzgerald, V. (eds.), Financiación del desarrollo y coherencia en las políticas de los donantes, Madrid, Los Libros de la Catarata, pp.181-211.

Hoffmeister, F. (2008): "InterPillar coherence in the European Union's civilian crisis management" en Blockmans, The European Union and Crisis Management - Policy and Legal Aspects, La Haya, T.M.C. Asser Press, pp.157-180.

MAEC (2013): IV Plan Director de la Cooperación Española 2013-2016, Secretaria General de Cooperación Internacional para el Desarrollo. Disponible en web: (http://www.ocud.es/es/Sala_de_prensa-Aprobado_IV_PD-ene13). Fecha de consulta: 08/01/2014.

Millán, N. (2010): "El caso de España”, en Alonso, J.A. (dir.), Coherencia de políticas para el desarrollo en cinco donantes del CAD: lecciones para el caso español, Madrid, Documento de trabajo número 42, pp. 145- 172.

Millán N. (2011): "Coherencia para el desarrollo en un mundo globalizado: más allá de las políticas de ayuda. Los casos de Suecia y España", Revista Sistema, 220, pp. 139- 161.

Millán, N. (2012): Transnacionalización del desarrollo y coherencia de políticas. Un análisis de los casos de España y Suecia, Tesis Doctoral.

Naciones Unidas. (2003): El Desarrollo Basado en un Enfoque de los Derechos Humanos: Hacia una Comprensión Colectiva entre las Agencias de las Naciones Unidas. Disponible en web: www.unfpa.org/derechos/docs/ entendimiento onu.doc. Fecha de consulta: 08/01/2014.

OCDE , (2000): "Government Coherence: The Role of the Centre of Government", Meeting of Senior Officials from Centres of Government on Government Coherence: the Role of the Centre of Government, Budapest, 6-7/10/2000.

OCDE, (2005): "Policy Coherence for Development. Promoting Institutional Good Practice", The Development Dimension Series, Paris, OECD.

OCDE, (2008): Synthesis Report on Policy Coherence for Development, Paris, Publications OCDE.

Oxfam, (2011): Cultivar un mundo mejor, Campaña CRECE. Disponible en web: http://www.oxfam.org/sites/www.oxfam.org/files/growing-a-better-future010611-es_0.pdf. Fecha de consulta: 08/01/2014. 
Piccioto, R. (2004): Institutional Approach to Policy Coherence for Development. Policy Coherence and Development Evaluation: Concepts, Issues and Possible Approaches, Paris, OECD Policy Workshop.

PNUD, (2010): Informe sobre Desarrollo Humano 2010. La verdadera riqueza de las naciones: Caminos al desarrollo humano, Madrid, Mundi-Prensa.

Portela, C. y K. Raube (2009): (In)Coherence in EU Foreign Policy: Exploring Sources and Remedies. Disponible en web: http://aei.pitt.edu/33122/1/portela._clara_\%282\%29.pdf. Fecha de consulta: $08 / 01 / 2014$.

Sanahuja, J.A. (2007): "La política española de cooperación a partir de 2008: el reto de culminar las reformas", Quórum. Revista de Pensamiento Iberoamericano, 19, pp. 37-55.

Sanahuja, J.A. y J. Schünemann (2012): "El nexo seguridad-desarrollo: Entre la construcción de la paz y la securitización de la ayuda" en Sanahuja, José Antonio (coord.), Construcción de la paz, seguridad y desarrollo. Visiones, políticas y actores, Madrid, Editorial Complutense. pp. 17-70.

Santos, B. de Sousa. (1997): "Hacia una concepción multicultural de los Derechos Humanos", Revista Análisis Político, 31, pp. 3-16.

Sen, A. (1999): Desarrollo y Libertad, Madrid, Planeta.

Stokke, O. (2003): "Coherencia de Políticas en la cooperación al desarrollo: compromisos, obstáculos, y oportunidades" en Alonso, J.A.y V. Fitzgerald (eds.), Financiación del desarrollo y coherencia en las políticas de los donantes, Madrid, Los Libros de la Catarata. pp. 181-211.

Unceta, K. (2009): "Desarrollo, subdesarrollo, maldesarrollo y posdesarrollo. Una mirada transdisclipinar sobre el debate y sus implicaciones", Carta Latinoamericana. Contribuciones en Desarrollo y Sociedad en América Latina, 7, pp. 1-34.

Unión Europea. (2007): EU Report on Policy Coherence for Development, Bruselas, Comisión Europea.

Zapata-Barrero, R. (2004): Multiculturalidad e inmigración, Madrid, Síntesis. 